\title{
Renormalization Group in the Local Potential Approximation
}

\author{
Giovanni Felder \\ Institut des Hautes Etudes Scientifiques, 35, route de Chartres, F-91440 Bures-sur-Yvette, \\ France
}

\begin{abstract}
In the local potential approximation, renormalization group equations reduce to a semilinear parabolic partial differential equation. We derive this equation and show the relation with the hierarchical model. We construct a family of non-trivial fixed points $u_{2 n}^{*}, n=2,3,4, \ldots$, which have the form of $n$-well potentials and exist in the ranges of dimensions $2<d<d_{n}=2+2 /(n-1)$. As $d \uparrow d_{n}, u_{2 n}^{*}$ tends to zero. For the Wilson fixed point $u_{4}^{*}$, we give bounds on critical exponents. In the case of dipole gas in this approximation we show that no non-trivial fixed points exist.
\end{abstract}

\section{Introduction}

Non-trivial fixed points of the renormalization group (RG) play a crucial role in the understanding of statistical mechanics systems in the vicinity of the critical point [1]. In the case of a symmetric scalar field (a classical statistical mechanics system with one-component order parameter) the non-trivial fixed points are expected to appear as bifurcating from the trivial massless fixed point as one varies continuously the dimension $d$ of space [2]. These bifurcations occur at the thresholds $d_{n}=2+2 /(n-1), n=2,3,4, \ldots$, where the linearized $\mathrm{RG}$ acquires a zero mode (see Fig. 1. The dotted lines represent branches which are believed to be unphysical). The fixed point relevant for three dimensional physics is given by extrapolating to $d=3$ the branch bifurcating at $4=d_{2}$ dimensions.

This pattern is not well understood from a rigorous point of view, but some pieces of it were established in toy models like Dyson's hierarchical one [3]: Bleher and Sinai [4] proved for this model the existence of a non-trivial fixed point if $d=d_{n}-\varepsilon$, where $\varepsilon>0$ is small enough. Their result was refined by Collet and Eckmann who proved that the $\varepsilon$-expansion is asymptotic [5]. Gawedzki and Kupiainen considered the case of an $N$-component spin system in the hierarchical approximation and constructed a fixed point in three dimensions for $N$ large enough [6]. Recently Witter and Koch [7] succeeded in constructing a fixed point 


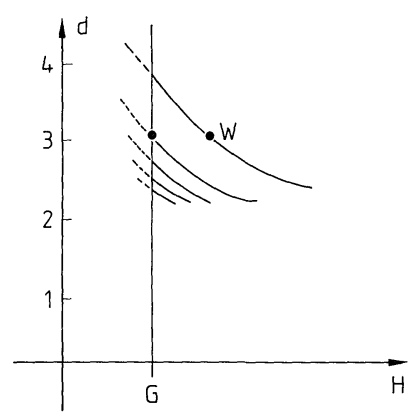

Fig. 1. Fixed points in the space of hamiltonians as functions of the dimension $d . G$ is the Gaussian, $W$ the Wilson fixed point

in three dimensions and $N=1$ for Gallavotti's [8] version of the hierarchical model with the help of a computer.

In this paper, we study a continuous scale version of the hierarchical model. It is given by the partial differential equation

$$
u_{t}=\frac{1}{2} u_{x x}-\frac{d-2}{2} x u_{x}+d u-\frac{1}{2} u_{x}^{2},
$$

describing the flow of the effective potential $u(t, x)$ on momentum scale $e^{-t}$ as a function of the field $x \in \mathbb{R}$. A similar equation was studied numerically by Hasenfratz and Hasenfratz [9] who found a non-trivial fixed point in three dimensions. Brydges and Kennedy [10] also studied similar equations in connection with the Mayer expansion.

Equation (1.1) can be derived in two ways: either as a limit of infinitesimal step of the recursion relation of the hierarchical model, or as the local potential approximation (LPA) to Wilson's "exact" renormalization group equations $[11,12]$. These derivations are given in Sect. 2.

The spectrum of the linearization at $u=0$ of (1.1) is

$$
\left\{\lambda_{n}=d-n(d-2) / 2, n=0,2, \ldots\right\}
$$

in

$$
L_{\text {even }}^{2}\left(e^{-\frac{d-2}{2} x^{2}} d x\right),
$$

and thus the bifurcation picture discussed at the beginning of this section should hold. Unfortunately conventional bifurcation analysis does not work here due to the nasty non-linearity, and we have to use other methods.

It turns out that RG fixed points must be identified with global stationary solutions of (1.1). Besides the trivial fixed points $u=0$ and $u=x^{2}-1 / d$ (high temperature fixed point), Eq. (1.1) has non-trivial global stationary solutions $u_{2 n}^{*}(x)$ with the form of $n$-well potentials in the ranges $2<d<d_{n}=2+2 /(n-1), n=2,3, \ldots$. These solutions converge pointwise to zero as $d \uparrow d_{n}$. Their large $x$ behaviour is dictated by (1.1), $u_{2 n}^{*}(x) \sim x^{2}$ as $x \rightarrow \pm \infty$, as one easily sees, making the ansatz $u(x)$ $\sim A x^{p}$ for $x \rightarrow \infty$. These results as well as some remarks on dynamics are contained in Sect. 3. Another equation which could be simpler to analyse as far as the 
dynamics is concerned is the LPA of the dipole gas. In the sine-Gordon representation the field $x$ of canonical dimension $\frac{d-2}{2}$ is replaced by a $d$-dimensional vector $x^{i}$ (the gradient of the field) with canonical dimension $\frac{d}{2}$. The
equation becomes

$$
u_{t}=\frac{1}{2} \Delta u-\frac{d}{2} x \cdot \nabla u+d u-\frac{1}{2}(\nabla u)^{2} .
$$

(here $d$ has to be taken integer). In this case the methods of Giga and Kohn [13] can be used to show that no non-trivial fixed points exist (Theorem 3.3).

The paper is organized as follows: in Sect. 2 we derive (1.1) from the LPA of Wilson's equation and from the hierarchical model as the scale factor $L \rightarrow 1$. In Sect. 3 , we discuss the equation and state the results. The $\varphi^{4}$ fixed point $u_{4}^{*}$ is constructed in Sect. 4, and the general construction of $u_{2 n}^{*}$ is in Sect. 5.

A short version of these results appeared in [14].

\section{Derivation of the Partial Differential Equation}

The P.D.E. we study in this paper can be understood in two ways: it is the local potential approximation to Wilson's continuous parameter renormalization group equation, and it is also the $L \rightarrow 1$ limit of the recursion relation of the hierarchical model, where $L$ is the scale parameter (the size of the block).

(a) The Local Potential Approximation. Renormalization group equations describe the change of the effective hamiltonian as the scale is varied. The effective hamiltonian can be written as a sum of the Gaussian fixed point and a potential, which is, in general, non-local. The local potential approximation (LPA) consists in projecting at each renormalization step the potential onto its local part. We describe this approximation in detail for Wilson's "exact renormalization group" equations [11]

$$
\begin{gathered}
\frac{\partial H}{\partial t}=\int_{q} h\left(q^{2}\right)\left[\frac{\delta H}{\delta \varphi_{q} \delta \varphi_{-q}}-\frac{\delta H}{\delta \varphi_{q}} \frac{\delta H}{\delta \varphi_{-q}}+\varphi_{q} \frac{\delta H}{\varphi_{q}}\right]+\int_{q}\left(\frac{d}{2} \varphi_{q}+q \nabla_{q} \varphi_{q}\right) \frac{\delta H}{\delta \varphi_{q}}, \\
h\left(q^{2}\right)=1-\frac{1}{2} \eta+2 q^{2},
\end{gathered}
$$

but the same result can be obtained from other RG schemes with continuous scale parameter, such as Polchinski's [15] (with a suitable cut-off function). The notation in (2.1) is the following: $H=H\left(t,\left\{\varphi_{q}\right\}\right)$ is the effective hamiltonian on scale $e^{-t}$ expressed as function of the Fourier components $\varphi_{q}$ of the field variable. The $2 \pi$-conventions are:

$$
\int_{q} \equiv \int \frac{d^{d} q}{(2 \pi)^{d}}, \quad \int_{q} \delta_{q-q_{0}} f(q)=f\left(q_{0}\right), \quad \frac{\delta \varphi_{q_{1}}}{\delta \varphi_{q_{2}}}=\delta_{q_{1}-q_{2}} .
$$

We neglect systematically $\varphi$ independent quantities on the right-hand side of (2.1), which only affect the zero point energy and are thus irrelevant to the computation of expectations. We set furthermore $\eta=0$ for consistency, since wave function renormalization is not present in this approximation. 
The perturbative treatment of (2.1) was developed by Wilson and Kogut [11] and Wegner [12]. We recall here some of their calculations: the Gaussian critical fixed point of (2.1) is, for $\eta=0$,

$$
H_{G}^{*}=\frac{1}{2} \int_{q} V_{2}^{*}(q) \varphi_{q} \varphi_{-q}, \quad V_{2}^{*}(q)=\frac{q^{2}}{q^{2}+e^{-2 q^{2}}} .
$$

The linearization of the generator of (2.1) at $H_{G}^{*}$,

$$
L=\int_{q} h\left(q^{2}\right) \frac{\delta^{2}}{\delta \varphi_{q} \delta \varphi_{-q}}+\int_{q}\left(\frac{d}{2} h\left(q^{2}\right)\left(1-2 V_{2}^{*}(q)\right)+q \nabla_{q} \varphi_{q}\right) \frac{\delta}{\delta \varphi_{q}},
$$

has the translation invariant eigenfunctions

$$
\mathcal{O}_{n r}=\int_{q_{1} \ldots q_{n}} P_{r}\left(q_{1}, \ldots, q_{n-1}\right): \prod_{i=1}^{n}\left(f\left(q_{i}^{2}\right) \varphi_{q_{i}}\right): \delta_{\substack{\sum_{i=1}^{n} q_{i} \\ i}}
$$

to the eigenvalues

$$
\lambda_{n r}=\frac{d-2}{2} n+|r|-d,
$$

where $r$ labels the homogeneous polynomials $P_{r}$, and $|r|$ is the degree of $P_{r}$. The function

$$
f\left(q^{2}\right)=\frac{e^{-q^{2}}}{q^{2}+e^{-2 q^{2}}}
$$

plays the role of the cut-off function in this approach. Wick-ordering is defined with respect to the covariance $V_{2}^{*}(q)^{-1}$ :

$$
: e^{i \int S_{q} \varphi_{-q}}:=e^{i \int S_{q} \varphi_{-q}+\frac{1}{2} \int S_{q} V_{2}^{*}(q)^{-1} S_{-q}} .
$$

The LPA is given by a projection of Eq. (2.1) onto the space spanned by the local operators $\mathcal{O}_{n 0}$. Acting on field monomials the projection operator is defined by taking the kernel at zero momentum:

$$
P \int_{q_{1} \ldots q_{n}} V\left(q_{1}, \ldots, q_{n-1}\right) \prod_{i=1}^{n} \varphi_{q_{i}} f\left(q_{i}^{2}\right) \delta_{\Sigma q_{i}}=V(0, \ldots, 0) \int \prod_{i=1}^{n} \varphi_{q_{i}} f\left(q_{i}^{2}\right) \delta_{\Sigma q_{i}} .
$$

The equation for the potential $V=H-H_{G}^{*}$ is

$$
\begin{aligned}
\frac{\partial V}{\partial t}= & \int_{q} h\left(q^{2}\right) \frac{\delta^{2} V}{\delta \varphi_{q} \delta \varphi_{-q}}+\int_{q}\left[\left(\frac{d}{2}+h\left(1-2 V_{2}^{*}\right)\right) \varphi_{q}+q \nabla_{q} \varphi_{q}\right] \frac{\delta V}{\delta \varphi_{q}} \\
& -\int h\left(q^{2}\right) \frac{\delta V}{\delta \varphi_{q}} \frac{\delta V}{\delta \varphi_{-q}} .
\end{aligned}
$$

To compute the LPA we assume that $V$ is in the range of $P$ and act with $P$ on the right-hand side of (2.9). The practical way of doing this is to expand $V$ into $\mathscr{O}_{n}$ 's:

$$
V=\sum \mu_{n} \mathcal{O}_{n}, \quad \mathcal{O}_{n}=\int \sum_{i=1}^{n}\left(\varphi_{i} f\left(q_{i}^{2}\right)\right) \delta_{\Sigma q_{i}}
$$


and compute the various terms explicitly:

$$
\begin{aligned}
P \int_{q} h\left(q^{2}\right) \frac{\delta^{2} \mathcal{O}_{n}}{\delta \varphi_{q} \varphi_{-q}} & =\left(\int_{q} h\left(q^{2}\right) f\left(q^{2}\right)^{2}\right) n(n-1) \mathcal{O}_{n-2}, \\
P \int_{q} g\left(q^{2}\right) \varphi_{q} \frac{\delta \mathcal{O}_{n}}{\delta \varphi_{q}} & =g(0) n \mathcal{O}_{n}, \\
P \int_{q} q \nabla_{q} \varphi_{q} \frac{\delta \mathcal{O}_{n}}{\delta \varphi_{q}} & =\left.P \frac{d}{d \lambda}\right|_{\lambda=1} \mathcal{O}_{n}\left(\varphi_{\lambda q}\right) \\
& =\left.P \frac{d}{d \lambda}\right|_{\lambda=1} \lambda^{-d(n-1)} \mathcal{O}_{n}\left(\varphi_{q}\right)=-d(n-1) \mathcal{O}_{n}\left(\varphi_{q}\right), \\
P \int h\left(q^{2}\right) \frac{\delta \mathcal{O}_{n}}{\delta \varphi_{q}} \frac{\delta \mathcal{O}_{m}}{\delta \varphi_{-q}} & =n \mathcal{O}_{n-1} m \mathcal{O}_{m-1},
\end{aligned}
$$

where (2.11c) was computed by scaling variables in the integral over $q$ in (2.10) and by using the fact that $f^{-1} \nabla_{q} f=0$ at $q=0$. We then identify $V\left(\left\{\varphi_{q}\right\}\right)=\sum_{n} \mu_{n} \mathcal{O}_{n}(\varphi)$ with the function in one variable $V(\varphi)=\sum_{n} \mu_{n} \varphi^{n}$ (the "density" of the local potential)

$$
\frac{\partial V}{\partial t}=A \frac{\partial^{2} V}{\partial \varphi^{2}}-\frac{d-2}{2} \varphi \frac{\partial V}{\partial \varphi}+d V-\left(\frac{\partial V}{\partial \varphi}\right)^{2}, \quad A=\int_{q} h\left(q^{2}\right) f\left(q^{2}\right)^{2}
$$

Equation (1.1) is obtained from (2.12) by the transformation

$$
V(t, \varphi)=A^{-1} u\left(t,(A / 2)^{1 / 2} \varphi\right) .
$$

(b) The L $\rightarrow 1$ Limit of the Hierarchical Model. The hierarchical model [3] in the version introduced by Gallavotti [8] reduces to the recursion relation which maps the effective potential on scale $\mu$ to the effective potential on scale $L^{-1} \mu$ given by

$$
\begin{gathered}
R_{L}: u(\mu, \cdot) \rightarrow u\left(L^{-1} \mu, \cdot\right), \\
u\left(L^{-1} \mu, x\right)=-\ln \int \exp \left[-L^{d} u\left(\mu, L^{1-d / 2} x+z\right)-z^{2} / 2(L-1)\right] \frac{d z}{\sqrt{2 \pi(L-1)}} .
\end{gathered}
$$

Here $x \in \mathbb{R}$ is the field (classical spin) variable. This recursion relation defines effective potentials on the discrete set of scales $L^{-k} \Lambda, k=0,1,2, \ldots$, where $\Lambda$ is some cut-off. In the limit $L \rightarrow 1$ this recursion relation becomes a differential equation

$$
\mu \frac{\partial u}{\partial \mu}=\lim _{L \rightarrow 1} \frac{u\left(L^{-1} \mu, x\right)-u(\mu, x)}{L^{-1}-1}=-\left.\frac{d}{d \lambda}\right|_{L=1}\left(R_{L} u\right)(\mu, x) .
$$

The factors $(L-1)$ in (2.13), (which are irrelevant as long as $L$ is a fixed number larger than one) were chosen so that the limit $L \rightarrow 1$ of the right-hand side of (2.13) is $u(\mu, x)$. Taking the derivative of the right-hand side of $(2.13)$ with respect to $L$ at $L=1$ is straightforward. The result is (1.1) in the variable $\mu=e^{-t}$ :

$$
-\mu \frac{\partial u}{\partial \mu}=\frac{1}{2} \frac{\partial^{2} u}{\partial x^{2}}-\frac{d-2}{2} x \frac{\partial u}{\partial x}+d u-\frac{1}{2}\left(\frac{\partial u}{\partial x}\right)^{2} .
$$


The reader may be confused by the fact that we did not impose a normalization condition on the zero point energy. This could be done by adding a Lagrange multiplier

$$
u_{t}=\frac{1}{2} u_{x x}-\frac{d-2}{2} x u_{x}+d u-\frac{1}{2} u_{x}^{2}+\theta(t)
$$

to enforce, e.g., the condition $u(t, 0)=0$. But solutions of $\left(1.1^{\prime}\right)$ are in a trivial one-toone correspondence with solutions of (1.1) obeying $u(0,0)=C$, for any fixed constant $C$. Indeed if $u$ is a solution of (1.1) with this property,

$$
\tilde{u}(t, x)=u(t, x)-u(t, 0), \quad \theta(t)=-u_{t}(t, 0)+d u(t, 0)
$$

is a solution of $\left(1.1^{\prime}\right)$. The inverse map is

$$
u(t, x)=\tilde{u}(t, x)+e^{d t} C-\int_{0}^{t} e^{d(t-s)} \theta(s) d s .
$$

\section{Discussion of the Equation, Results}

We begin this section by discussing some elementary properties of the partial differential equation derived in the previous section. The conclusion will be that non-trivial fixed points must be identified with the global stationary solutions of (1.1). We then state our main result (Theorem 3.2) and conclude with some remarks and further results.

At first sight, the fixed point equation

$$
u^{\prime \prime}(x)-(d-2) x u^{\prime}(x)+2 d u(x)-u^{\prime}(x)^{2}=0
$$

has a lot of solutions, parametrized by the initial condition $u(0)\left[u^{\prime}(0)=0\right.$ if $u$ is even]. Note however that (3.1) admits solutions that blow up (like $\left.-\ln \left|x-x_{0}\right|\right)$ at finite value $x_{0}$ of the field variable $x$. A solution of (3.1) going to infinity at $x_{0}$ corresponds to a Gibbs factor $p=\exp (-u)$ which vanishes at $x_{0}$. The following theorem states that all fixed points which are limits for $t \rightarrow \infty$ of effective Gibbs factors $\varrho(x, t)=\exp (-u(x, t))$ with $\varrho(x, 0)>0$ are positive. Thus the relevant fixed points are global solutions of (3.1).

Theorem 3.1. (i) Let $T>0, d>2$, and let $\varrho(t, x)$ be a bounded classical solution on $[0, T] \times \mathbb{R}$ of

$$
\varrho_{t}=\frac{1}{2} \varrho_{x x}-\frac{d-2}{2} x \varrho_{x}+d \varrho \ln |\varrho|
$$

with positive initial data $\varrho(0, x)$. Then $\varrho(t, x)$ is positive.

(ii) Let $Q^{*}$ be a bounded non-negative stationary $C^{\prime \prime}(\mathbb{R})$ solution of (3.2). Then either $\varrho^{*}$ vanishes identically or $\varrho^{*}(x)>0$ for all $x \in \mathbb{R}$.

Proof. (i) Let $\varrho=e^{(d-2) x^{2} / 4} g$; then $g$ obeys the equation

$$
g_{t}=\frac{1}{2} g_{x x}-q(x) g, \quad q(x)=\frac{1}{2}\left(\frac{d-2}{2}\right)^{2} x^{2}-\frac{d-2}{4}-d \ln |\varrho| .
$$


Since $\varrho$ is bounded, $g$ is $L^{2}$ and the "potential" $q$ is bounded below. The claim follows using the Feynman-Kac formula.

(ii) Suppose the claim is false. Then there exists an $x_{0} \in \mathbb{R}$ and a $\delta>0$ such that $\varrho^{*}\left(x_{0}\right)=0$, and $\varrho^{*}(x)>0$ for $x_{0}-\delta<x<x_{0}$ [or for $x_{0}<x<x_{0}+\delta$ but the latter case can be reduced to the former by noting that if $\varrho^{*}(x)$ is a solution, so is $\left.\varrho^{*}(-x)\right]$. Since $\varrho^{*}$ is, by assumption, nonnegative, $\varrho^{* \prime}$ must vanish at $x_{0}$. Let $r^{2}(x)=\varrho^{*}(x)^{2}$ $+\varrho^{*^{\prime}}(x)^{2}$. By continuity $r(x) \rightarrow 0$ as $x \uparrow x_{0}$. We show that this is not possible: $r$ obeys

$$
r r^{\prime}=(d-2) x\left(\varrho^{* \prime}\right)^{2}+\varrho^{*} \varrho^{* \prime}\left(2 d \ln \frac{1}{\varrho^{*}}+1\right) .
$$

For $\varrho>0$ small enough $\varrho \ln (1 / \varrho)$ is positive and increasing in $\varrho$. Estimate the righthand side of (3.4) by using $\varrho^{*} \leqq r,\left|\varrho^{* \prime}\right| \leqq r$, and, if $\delta^{\prime}$ is small enough, $\varrho^{*} \ln \left(1 / \varrho^{*}\right)$ $\leqq r \ln (1 / r)$, for $x_{0}-\delta^{\prime}<x<x_{0}$,

$$
r^{\prime} \geqq-(d-2)\left|x_{0}-\delta^{\prime}\right| r-r\left(2 d \ln \frac{1}{r}+1\right)=-r 2 d \ln \frac{D}{r}, \quad D>1 .
$$

We get thus the estimate on $r\left(x_{0}\right)$ in terms of $r\left(x_{0}-\delta^{\prime}\right)$,

$$
r\left(x_{0}\right) \geqq D \exp \left(e^{2 d \delta^{\prime}} \ln \frac{r\left(x_{0}-\delta^{\prime}\right)}{D}\right) .
$$

Thus $r\left(x_{0}\right)>0$ which is a contradiction.

We are now ready to state our results. The proofs are contained in the next two sections.

Theorem 3.2. Let $2<d<d_{n}=2+\frac{2}{n-1}, n=2,3,4, \ldots$. Then there exists an even fixed point $u_{2 n}^{*}$, the " $\phi^{2 n}$ fixed point", (i.e. an even global solution of (3.1)). The function $u_{2 n}^{*}(x)$ has $2 n-1$ critical points and grows to infinity as $x \rightarrow \pm \infty$.

This picture of " $n$-well potentials" is in agreement with (the lowest order) $\varepsilon$-expansion which predicts the existence of fixed points

$$
u_{2 n}^{*}(x)=c_{n} \varepsilon H_{2 n}\left((d / 2-1)^{1 / 2} x\right)+O\left(\varepsilon^{2}\right) \text { for } d=d_{n}-\varepsilon,
$$

where $H_{2 n}$ is the $2 n^{\text {th }}$ Hermite polynomial.

We cannot establish that the $\varepsilon$-expansion is asymptotic, but we can show that, as $\varepsilon \rightarrow 0^{+}, u_{2 n}^{*}(x) \rightarrow 0$ pointwise in $x$. We defer the proof of this fact (for $2 n=4$ ) to the end of Sect. 4. Of course, no stronger convergence to zero is expected since the large $x$ asymptotics of $u_{2 n}^{*}$ is $x^{2}$ as dictated by (3.1).

A numerical study around $d=4$ shows that the $\varepsilon$-expansion is accurate [in the sense that the initial condition $u_{4}^{*}(0)$ predicted by the $\varepsilon$-expansion does correspond to a global solution] for $\varepsilon>0$. No fixed points corresponding to $\varepsilon<0$ seem to exist.

Critical exponents are given in terms of eigenvalues of the linearized flow equation at the non-trivial fixed point. The information contained in Theorem 3.2 is sufficient to give some (rough) bound on these eigenvalues: consider the renormalization group equation linearized around the Wilson fixed point $u_{4}^{*}$ in $2<d<4$ dimensions:

$$
\Delta \dot{u}=L \Delta u, \quad L=\frac{1}{2} \frac{d^{2}}{d x^{2}}-\left(\frac{d-2}{2} x+u_{4}^{* \prime}\right) \frac{d}{d x}+d ;
$$


$L$ is selfadjoint in $L^{2}\left(\mathbb{R}, e^{-\frac{d-2}{2} x^{2}-2 u_{4}^{*}(x)} d x\right)$. The obvious eigenfunctions 1 to the eigenvalue $\lambda_{0}=d$ (corresponding to a trivial rescaling of the zero point energy) and $u_{4}^{* \prime}$ to the eigenvalue $\lambda_{3}=(d-2) / 2$ have, respectively, no zero and three zeros. Therefore there exists an odd eigenfunction with one zero and eigenvalue $\lambda_{1}$ and an even eigenfunction with two zeros and eigenvalue $\lambda_{2}$ such that

$$
d=\lambda_{0}>\lambda_{1}>\lambda_{2}>\lambda_{3}=\frac{d-2}{2} \text {. }
$$

[The oscillation theorem holds since $L$ can be represented as minus a Schrödinger operator on $L^{2}(\mathbb{R}, d x)$.] The critical exponents of the correlation length, susceptibility and magnetization

$$
\xi \sim\left(T-T_{c}\right)^{-v}, \quad \chi \sim\left(T-T_{c}\right)^{-\gamma}, \quad M\left(T_{c}\right) \sim h^{1 / \delta}
$$

are given by ( $\eta=0$ in our approximation)

$$
v=\frac{1}{\lambda_{2}}, \quad \gamma=2 v, \quad \delta=\frac{2 \lambda_{1}}{d-2},
$$

and obey the bounds

$$
\frac{1}{d}<v<\frac{2}{d-2}, \quad 1<\delta<\frac{2 d}{d-2}
$$

Unfortunately, our control on the P.D.E. is not sufficient to say something about the flow in the vicinity of the fixed points beyond the linear approximation, or to construct the basin of attraction of the fixed point. A simple argument, though, gives a necessary condition for a potential to be in the basin of attraction of a fixed point $u_{2 n}^{*}$. In fact, for "generic" solutions $u(t, x)$ of (1.1), the number of critical points

$$
n(t)=\left\{x \mid u_{x}(t, x)=0\right\}
$$

is a non-increasing function of $t$. To see this consider the set

$$
C=\left\{(t, x) \in \mathbb{R}_{+} \times \mathbb{R} \mid u_{x}(t, x)=0\right\},
$$

and let $v(t, x)=u_{x}(t, x)$. We make the "genericity" assumption that $\left(v_{t}, v_{x}\right) \neq(0,0)$ on $C$. Then $C$ is a collection of curves, locally represented by functions $x(t)$ or $t(x)$. If, at time $t_{0}, n(t)$ jumps, there exists a point $x_{0}$ such that $t_{0}=t\left(x_{0}\right)$ and $t^{\prime}\left(x_{0}\right)$ $=-v_{x}\left(t_{0}, x_{0}\right) / v_{t}\left(t_{0}, x_{0}\right)=0$ and if $n(t)$ increases, $t^{\prime \prime}\left(x_{0}\right) \geqq 0$. The derivative of (1.1)

$$
v_{t}=\frac{1}{2} v_{x x}-\frac{d-2}{2} x v_{x}+\frac{d+2}{2} v-v v_{x}
$$

gives that

$$
t^{\prime \prime}\left(x_{0}\right)=-v_{x x}\left(t_{0}, x_{0}\right) / v_{t}\left(t_{0}, x_{0}\right)=-2<0 .
$$

Thus $n(t)$ cannot increase.

Actually, we cannot expect the condition $\left(v_{t}, v_{x}\right) \neq 0$ on $C$ to hold for generic even solutions since at $x=0$ by symmetry $v=v_{t}=0$ for all $t$. We can weaken this 
condition by requiring $\left(v_{t} / x,(v / x)_{x}\right) \neq(0,0)$ on $C^{*}=\{(t, x) \mid v(t, x) / x=0\}$. For $x \neq 0$ this is equivalent to the previous condition. If a new pair of zeros appears at $x=0$, $C^{*}$ can be locally described by a function $t(x)$ with $t^{\prime}(0)$ and $t^{\prime \prime}(0) \geqq 0$. But

$$
t^{\prime \prime}(0)=\lim _{x \rightarrow 0}-\frac{(v / x)_{x}}{v_{t} / x}=-\frac{1}{3} \frac{v_{x x x}(0)}{v_{t x}(0)},
$$

and taking the partial derivative with respect to $x$ of (3.6) yields $t^{\prime \prime}(0)=-2 / 3<0$, a contradiction. We conclude that, generically, a necessary condition for a function $u(0, x)$ to be in the basin of attraction of $u_{2 n}^{*}$ is that it has at least $2 n-1$ critical points.

We conclude by stating a result on the dipole gas case (1.2) about non-existence of fixed point. We use a method introduced by Giga and Kohn [13] to study selfsimilar blowing up solutions of a non linear heat equation.

Theorem 3.3. Let u be a $C^{\prime \prime}$ global stationary solution of (1.2) which is bounded below. Then $u$ vanishes identically.

Proof. Let $\varrho=\exp \left[-u\left((2 / d)^{1 / 2} x\right)\right]$. If $u$ is a solution of $(1.2)$ with $u_{t}$ replaced by 0 , $\varrho$ is a positive solution of

$$
\nabla(\alpha \nabla \varrho)+2 \alpha \varrho \ln \varrho=0,
$$

where

$$
\alpha(x)=e^{-x^{2} / 2} .
$$

The key step is to use the identities

$$
\begin{aligned}
& d \int(\nabla \varrho)^{2} \alpha d x-2 d \int \varrho^{2} \ln \varrho \alpha d x=0, \\
& \int\left(d-x^{2}\right) \varrho^{2} \alpha d x-\int x^{2}(\nabla \varrho)^{2} \alpha d x+2 \int x^{2} \varrho^{2} \ln \varrho \alpha d x=0, \\
& \left.\int\left(d+x^{2}\right)(\nabla \varrho)^{2} \alpha d x\right)-\int\left(d-x^{2}\right) \varrho^{2} \alpha d x+2 \int\left(d-x^{2}\right) \varrho^{2} \ln \varrho \alpha d x=0,
\end{aligned}
$$

which are obtained by multiplying (3.7) by $\varrho, x^{2} \varrho$, and $x \cdot \nabla \varrho$, respectively, and integrating over $\mathbb{R}^{d}$. Summing up the three identities we get

$$
\int(\nabla \varrho)^{2} \alpha d x=0 .
$$

Thus $\varrho$ is constant and by (3.7) equal to one.

This "miraculous cancellation" is peculiar to the borderline case of Eq. (1.2): it depends crucially on the ratio between the coefficient of $u$ and the coefficient of $x \cdot \nabla u$ being -2 . A similar property is shared by the Giga-Kohn equation.

\section{The Non-Trivial $\varphi^{4}$ Fixed Point}

This section is devoted to the construction of the non-trivial fixed point $u_{4}^{*}$ (the $\varphi^{4}$ fixed point) in $2<d<4$ dimensions. As pointed out in Sect. 3 , a fixed point is a global solution of the ordinary differential equation

$$
u^{\prime \prime}(x)-(d-2) x u^{\prime}(x)+2 d u(x)-u^{\prime}(x)^{2}=0 .
$$


It is convenient to take the derivative of this equation and study the equation for $v(x)=u^{\prime}(x)$ :

$$
v^{\prime \prime}-(d-2) x v^{\prime}+(d+2) v-2 v v^{\prime}=0 .
$$

After the rescaling $v \rightarrow \sqrt{d+2} v(\sqrt{d+2} \cdot)$, the equation is reduced to

$$
v^{\prime \prime}-\frac{x}{\sigma} v^{\prime}+v-2 v v^{\prime}=0, \quad \sigma=\frac{d+2}{d-2},
$$

which we view as a dynamical system on the phase plane ( $x$ is "time"):

$$
\begin{aligned}
v^{\prime} & =w, \\
w^{\prime} & =\frac{x}{\sigma} w-v+2 v w .
\end{aligned}
$$

It is an easy exercise to solve this equation in closed form in the limit $\sigma \rightarrow \infty(d \rightarrow 2)^{1}$, but if $d>2$ the system is non-autonomous, and more difficult to handle. It is sufficient to construct a global solution $(v(x), w(x))$ for $x \geqq 0$, since the continuation for negative $x$ is given by

$$
(v(-x), w(-x))=(-v(x), w(x)),
$$

provided the initial condition is such that $v(0)=0$, (we restrict ourselves with even potentials). Divide the plane into six regions I, II, .., VI (Fig. 2)

$$
\begin{array}{cl}
\mathrm{I}=\left\{(v, w) \mid w \geqq \frac{1}{2}, v<0\right\}, & \mathrm{II}=\left\{(v, w) \mid w \geqq \frac{1}{2}, v \geqq 0\right\}, \\
\mathrm{III}=\left\{(v, w) \mid 0 \leqq w<\frac{1}{2}, v<0\right\}, & \mathrm{IV}=\left\{(v, w) \mid 0<w<\frac{1}{2}, v \geqq 0\right\}, \\
\mathrm{V}=\{(v, w) \mid w<0, v \leqq 0\}, & \mathrm{V}=\{(v, w) \mid w \leqq 0, v>0\} .
\end{array}
$$

The arrows in Fig. 2 show the direction of the vector field (for $x>0$ ), on the boundaries of these regions: one sees that these boundaries can be crossed in only one way by solutions of (4.4). We show more: the solutions leave all regions except IV within finite time.

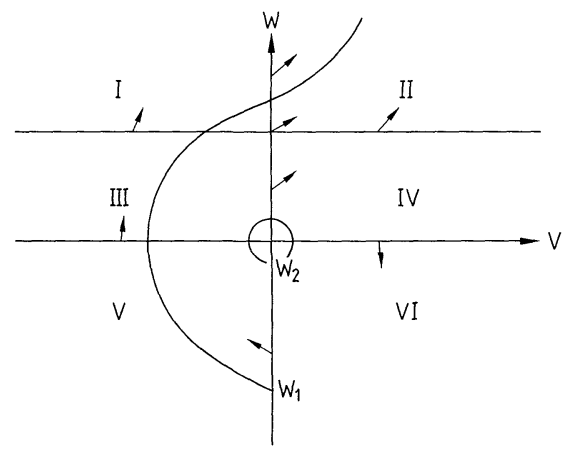

Fig. 2. The partition of the phase plane in six regions

${ }^{1}$ The $\sigma=\infty$ trajectories are given by the equation $\left(w-\frac{1}{2}\right) \exp \left(2\left(w-v^{2}\right)\right)=J, J \geqq-\frac{1}{2}$. They are closed for $-\frac{1}{2} \leqq J<0$ and blow up at finite $x$ for $J>0$. The trivial fixed points correspond to $J=-\frac{1}{2}, 0$ 
Proposition 4.1. Let $1<\sigma<\infty, x_{0}>0$, and $\left(v_{0}, w_{0}\right) \in A=\mathrm{I}$, II, III, V or VI. Then there exists a time $x_{1}>x_{0}$ (depending continuously on $\sigma, x_{0}, v_{0}, w_{0}$ ) such that if $(v(x), w(x)$ ) is the solution of (4.4) with $v\left(x_{0}\right)=v_{0}$ and $w\left(x_{0}\right)=w_{0},\left(v\left(x_{1}\right), w\left(x_{1}\right)\right.$ is in a region $B \in\{\mathrm{I}, \ldots, \mathrm{VI}\}$ such that there is an arrow from $A$ to $B$ in the following diagram:

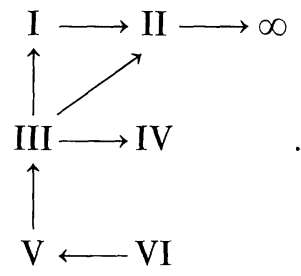

The arrow from II to $\infty$ indicates that all solutions going through II, and only these, blow up in finite time.

Proof. We prove all statements represented by (4.6) separately calling $v\left(x_{0}\right)=v_{0}$, $w\left(x_{0}\right)=w_{0}$.

(a) $\mathrm{I} \rightarrow$ II. In region I we have $v^{\prime} \geqq \frac{1}{2}, w^{\prime} \leqq \frac{x}{\sigma} w$. Thus, as long as $(v, w) \in \mathrm{I}, v(x) \geqq v_{0}$ $+\frac{1}{2}\left(x-x_{0}\right), w \leqq w_{0} \exp \left[\left(x^{2}-x_{0}^{2}\right) / \sigma\right]$, implying that the solution does not blow up in I and that after a time at most $-2 v_{0},(v, w)$ enters II.

(b) II $\rightarrow \infty$. In II, $v^{\prime} \geqq \frac{1}{2}$, thus $v \geqq \frac{1}{2}\left(x-x_{0}\right)$ and, if $x$ is larger than $x_{0}+\sqrt{2 \sigma}, x v \geqq \sigma$. Let us set, for later purposes, $x_{0}^{\prime}=\max \left(x_{0}+\sqrt{2 \sigma}, \sqrt{2} \sigma\right)$. Now either (i) $w\left(x_{0}^{\prime}\right)$ $>v\left(x_{0}^{\prime}\right)^{2}$ or (ii) $w\left(x_{0}^{\prime}\right) \leqq v\left(x_{0}^{\prime}\right)^{2}$. In case (i), $w(x)>v(x)^{2}$ for all $x \geqq x_{0}^{\prime}$ since, if $w(x) \geqq v(x)^{2}$,

$$
\frac{d}{d x}\left(w-v^{2}\right)=\frac{x}{\sigma} w-v \geqq\left(\frac{x}{\sigma} v-1\right) v \geqq 0 .
$$

Hence $v^{\prime}(x)>v^{2}(x)$ for $x>x_{0}^{\prime}$ with escape to infinity in finite time. In the second case, (ii), either at some $x_{0}^{\prime \prime}, w\left(x_{0}^{\prime \prime}\right)>v^{2}\left(x_{0}^{\prime \prime}\right)$ and we are back to case (i), or $w(x) \leqq v^{2}(x)$ for all $x>x_{0}^{\prime}$. Then using (4.4) and the fact that $w \geqq \frac{1}{2}$ in II, we get

$$
w^{\prime} \geqq \frac{x}{\sigma} w+(2 w-1) \sqrt{w} \geqq\left(\frac{x}{\sqrt{2} \sigma}-1\right) \sqrt{w}+2 w^{3 / 2} \geqq 2 w^{3 / 2}
$$

since, by assumption, $\frac{x}{\sqrt{2} \sigma} \geqq 1$; the solution blows up again.

(c) III $\rightarrow$ I, III $\rightarrow$ II, III $\rightarrow$ IV. In III, $v^{\prime} \geqq 0$ and solutions cannot blow up in this region. The bound $w^{\prime} \geqq \frac{x}{\sigma} w$ implies that, if $w_{0}>0, w(x) \geqq w_{0} \exp \left(x^{2}-x_{0}^{2}\right) / 2$, and the solution leaves III in finite time. If $w_{0}=0$, the bound $w^{\prime} \geqq-v>0$ implies that for $x_{0}^{\prime}>x_{0}, w\left(x_{0}^{\prime}\right)>0$ and the previous argument can be used.

(d) $\mathrm{V} \rightarrow$ III. We only prove the statement for $x_{0}=0$, which is all we need to construct the $\varphi^{4}$ fixed point. The general case will be proven in Sect. 5. Let

$$
S_{x}=\{(v, w) \mid I(x, v, w) \geqq 0\} \cap V, \quad I(x, v, w)=\frac{x w}{1-2 w}-v .
$$


We claim:

(i) For $x=0, S_{x}=V$.

(ii) If $(v(x), w(x)) \in V$ for $x \in\left[x_{1}, x_{2}\right]$ and $\left(v\left(x_{1}\right), w\left(x_{1}\right)\right) \in S_{x_{1}}$ then $(v(x), w(x)) \in S_{x}$ for all $x \in\left[x_{1}, x_{2}\right]$.

(iii) All solutions $(v(x), w(x)) \in S_{x}$ enter III after a finite time.

These properties immediately imply V $\rightarrow$ III, if $x_{0}=0$. Proof of (i), (ii), (iii): (i) is obvious, (iii) follows from the fact that, in $S_{x}$,

$$
w^{\prime}=\sigma^{-1}(1-2 w) I(x, v, w)-\left(1-\sigma^{-1}\right)(1-2 w) v \geqq\left(1-\sigma^{-1}\right) v .
$$

If $\left(v\left(x_{1}\right), w\left(x_{1}\right)\right) \in S_{x_{1}}$ for some $x_{1}>0, v\left(x_{1}\right) \leqq \frac{x_{1} w\left(x_{1}\right)}{1-2 w\left(x_{1}\right)}<0$, and, if $(v(x), w(x))$ is a solution in $S_{x}, v(x) \leqq v\left(x_{1}\right)$, since $v^{\prime}=w<0$, and from (4.10), $w^{\prime} \geqq\left(1-\sigma^{-1}\right)\left|v\left(x_{1}\right)\right|$ and $w$ becomes positive within finite time. No escape to infinity is possible in this region since by (4.10) $w^{\prime} \geqq 0$, so that $w$, and thus $v^{\prime}$, are bounded below by $w_{0}$. To prove (ii), we show that $I(x, v(x), w(x))$ is non-decreasing in $x$ as long as $(v(x), w(x)) \in S_{x}$ :

$$
\frac{d}{d x} I(x, v(x), w(x))=\frac{2 w^{2}}{1-2 w}+\frac{x w^{\prime}}{(1-2 w)^{2}} \geqq 0,
$$

by (4.10).

(e) VI $\rightarrow \mathrm{V}$. In VI, $v>0, w \leqq 0$. Thus, by (4.4), $v^{\prime} \leqq 0$, and $w^{\prime}<0$ for $x \geqq x_{0}$, and $(v, w)$ a solution in $\mathrm{VI}$,

$$
v(x) \leqq v_{0}, \quad w(x)<w_{0} .
$$

These bounds can be inserted again in (4.4) to yield

$$
v^{\prime}(x) \leqq w_{0}, \quad w^{\prime}(x) \geqq \frac{x}{\sigma} w(x)-v_{0}(1-2 w(x)) .
$$

The second (linear) bound implies that no solution can blow up in VI, and the first that $v(x)$ becomes negative within finite time, provided $w_{0}<0$. If $w_{0}=0$, we can use the bound $w^{\prime} \leqq-v$, to show that for $x_{0}^{\prime}>x_{0}, w\left(x_{0}^{\prime}\right)<0$, and the argument applies.

The next step is to construct two solutions, one of which blows up without crossing the positive $v$ axis and one going once around the origin and crossing the positive $v$ axis. These two solutions are drawn on Fig. 2.

Proposition 4.2 (Construction of $w_{1}$ ). Let $(v(x), w(x)) x \geqq 0$ be a solution of (4.4) with initial condition $\left(0, w_{0}\right)$ at $x=x_{0} \geqq 0$. There exists a constant $w_{1}=w_{1}(\sigma)<0$ such that if $w_{0} \leqq w_{1},(v(x), w(x))$ enters region II (and thus blows up) in a finite time $\bar{x}_{1}$ going successively through regions $\mathrm{V}, \mathrm{III}, \mathrm{I}$.

Proof. Note first that $J(v, w)=\left(w-\frac{1}{2}\right) \exp \left(2\left(w-v^{2}\right)\right)$ is an increasing Liapunov function. Indeed,

$$
\frac{d}{d x} J(v(x), w(x))=\frac{2 x}{\sigma} w^{2} e^{2\left(w-v^{2}\right)} \geqq 0, \quad x \geqq 0,
$$

as can be checked by explicit calculation. Its level sets are the trajectories of the limiting equation $\sigma \rightarrow \infty$. The solution $(v, w)$ with initial condition $\left(0, w_{0}\right)$ enters III 
after finite time. Thus there exists an $x_{1}>0$ such that $w\left(x_{1}\right)=0$ and $v\left(x_{1}\right)<0$. Since $J$ is non-decreasing,

$$
J\left(v\left(x_{1}\right), 0\right) \geqq J\left(0, w_{0}\right), \quad v\left(x_{1}\right)^{2} \geqq\left|w_{0}\right|-\frac{1}{2} \ln \left(1+2\left|w_{0}\right|\right) .
$$

Thus $v\left(x_{1}\right)$ can be made as negative as desired by choosing $\left|w_{0}\right|$ large enough. In region III, $v^{\prime}=w \leqq \frac{1}{2}$, hence, for $x \geqq x_{1}, v(x) \leqq v\left(x_{1}\right)+\frac{1}{2}\left(x-x_{1}\right)$. We consider times $x$ such that

$$
x-x_{1} \leqq\left|v\left(x_{1}\right)\right|
$$

For these times

$$
w^{\prime} \geqq \frac{x}{\sigma} w-v_{1}\left(\frac{1}{2}-w\right), \quad v_{1} \equiv v\left(x_{1}\right)
$$

As long as $w \leqq \frac{1}{4}$, we use the second term on the right-hand side of (4.17):

$$
w^{\prime} \geqq \frac{1}{4}\left|v\left(x_{1}\right)\right|, \quad w(x) \geqq\left|v_{1}\right| \frac{1}{4}\left(x-x_{1}\right),
$$

so that after a time at most $\frac{1}{\left|v_{1}\right|}, w(x) \geqq \frac{1}{4}$. For

$$
\frac{1}{2} \geqq w(x) \geqq \frac{1}{4} \quad \text { and } \quad x \geqq \frac{1}{\left|v_{1}\right|}+x_{1} \equiv x_{2},
$$

we use the first term in (4.17):

$$
w^{\prime} \geqq \frac{x}{4 \sigma}, \quad w \geqq \frac{1}{4}+\frac{1}{4 \sigma}\left(x^{2}-x_{2}^{2}\right),
$$

and $w(x) \geqq \frac{1}{2}$ for some $x \leqq x_{3} \equiv x_{1}+\frac{1}{\left|v_{1}\right|}+\sqrt{\sigma}$, and the solution enters I, provided the condition (4.16) is fulfilled for $x=x_{3}$, which is the case if $v\left(x_{1}\right)$ is chosen large enough.

Proposition 4.3 (Construction of $w_{2}$ ). Let $3<\sigma<\infty$ (i.e. $2<d<4$ ) and let $(v(x), w(x)), x \geqq 0$ be a solution of (4.4) with initial conditions $\left(0, w_{0}\right)$ at $x=0$. There exist a constant $w_{2}=w_{2}(\sigma)<0$, such that if $0>w_{0} \geqq w_{2},(v, w)$ enters region VI in a finite time $\bar{x}_{2}$, going successively through regions $\mathrm{V}$, III, IV.

Proof. Consider first the linear approximation to (4.4):

$$
v^{\prime \prime}-\frac{x}{\sigma} v^{\prime}+v=0
$$

We prove in the appendix that the odd solution of this equation has, for $\sigma>3$ and $x>0$, at least one zero and at least two critical points. Let $x_{1}$ be the smallest positive zero and $y_{1}, y_{2}$ be the smallest positive critical points, then $0<y_{1}<x_{1}<y_{2}$. If the initial condition $w_{0}=v^{\prime}(0)$ is negative, the solution of (4.20) enters VI in time $y_{2}+\delta$ going through the path described in the proposition. Choose then $\left|w_{2}\right|$ so small that the solution of the non-linear equation is so close to the solution of the linear equation (4.20) up to time $y_{2}+\delta$ that it also has the property of entering VI after visiting V, III, IV. 
The next step is the actual construction of the fixed point $\left(v^{*}, w^{*}\right)$. It is a solution with initial condition lying between $w_{2}$ and $w_{1}$, which goes (for $x \geqq 0$ ) through regions V, III to region IV, and stays there forever. In IV $v^{\prime}=w \leqq \frac{1}{2}$, thus no blow up in this region is possible and the solution is global. The function $v^{*}(x)$, $x \in \mathbb{R}$, has three zeros so that the corresponding solution of (4.1),

$$
u^{*}(x)=\int_{0}^{x} \sqrt{d+2} v^{*}(\sqrt{d+2} y) d y-\frac{d+2}{2 d} w^{*}(0)
$$

has three critical points. The construction of $\left(v^{*}, w^{*}\right)$ relies on the so-called BleherSinai [4] argument: to formulate it in a clear way we first modify (4.4) in the regions II and VI. Since the goal is to construct a solution that does not enter these regions, it will not matter how the equation looks like there. The modified equation is

$$
\begin{gathered}
v= \begin{cases}w, & \text { if } \\
0, & \text { if } \quad(v, w) \notin \mathrm{VI}, w) \in \mathrm{VI},\end{cases} \\
w^{\prime}= \begin{cases}\frac{x}{\sigma} w-v+2 v w, & \text { if } \quad(v, w) \notin \mathrm{II}, \\
\frac{x}{\sigma} w, & \text { if } \quad(v, w) \in \mathrm{II} .\end{cases}
\end{gathered}
$$

Note that the vector field (4.21) is continuous except for the negative $w$ axis. This is not a problem since no solutions of (4.21) cross this axis. Equation (4.21) has the two following properties:

(i) All solutions $(v(x), w(x))$ of (4.21) exist for all $x>0$.

(ii) For all $w_{1}<w_{2}<0$ there exists an $\bar{x}_{3}>0$ such that all solutions $(v(x), w(x))$ of (4.21) with $v(0)=0, w_{1}<w(0)<w_{2}$, have the property that $v(x)>0$ for $x>\bar{x}_{3}$.

Property (i) follows from the fact (Proposition 4.1) that only solutions going through II can escape to infinity in finite time. In this region (4.21) is linear and no escape is possible. Again by Proposition 4.1, the solutions considered in (ii) enter after a finite time $x_{1}$ region II or region IV. The vector field was modified in such a way that VI is invariant and no return to $v<0$ is possible and (ii) holds. Any other modification of (4.4) in VI and II with (i), (ii) can be used for the following argument. The construction of $\left(v^{*}, w^{*}\right)$ is contained in

Proposition 4.4. Let $3<\sigma<\infty$ (i.e. $2<d<4$ ). There exist $a w_{0}<0$ and times $0<x_{1}<x_{2}$ such that the solution $(v(x), w(x)), x \geqq 0$ of (4.21) with initial conditions $v(0)=0, w(0)=w_{0}$, obeys

$$
\begin{gathered}
(v, w) \in \mathrm{V}, \quad 0 \leqq x<x_{1}, \\
(v, w) \in \mathrm{III}, \quad x_{1} \leqq x<x_{2}, \\
(v, w) \in \mathrm{IV}, \quad x \geqq x_{2},
\end{gathered}
$$

and is thus a solution of (4.4) as well.

Proof. We consider the interval of initial conditions $I_{0}=\left[w_{1}, w_{2}\right]$, where $w_{1}, w_{2}$ are the constants introduced in Propositions 4.2, 4.3. Let, for $x>0, \phi_{x, x_{0}}$ denote the flow generated by (4.21). Choose $x>\max \left(\bar{x}_{1}, \bar{x}_{2}, \bar{x}_{3}\right)$, where $\bar{x}_{1}, \bar{x}_{2}, \bar{x}_{3}$ are defined in 
Proposition 4.2, 4.3 and (ii), respectively. From (ii) we know that $\phi_{x, 0}\left(0 \times I_{0}\right)$ is a continuous curve lying in $\{v>0\}$. Moreover, by Proposition $4.2, \phi_{x, 0}\left(0, w_{1}\right) \in$ II and, by Proposition $4.3, \phi_{x, 0}\left(0, w_{2}\right) \in \mathrm{VI}$. Thus there exists a piece $P Q$ of the curve $\phi_{x, 0}\left(0 \times I_{0}\right)$ lying in IV such that $P \in\left\{(v, w) \mid v>0, w=\frac{1}{2}\right\}$ and $Q \in\{(v, w) \mid v>0, w=0\}$ (see Fig. 2). Correspondingly there exists a closed interval $I_{1}=\left[w_{1}^{(1)}, w_{2}^{(1)}\right] \subset I_{0}$, such that $\phi_{x, 0}\left(0 \times I_{1}\right)=P Q$. Consider now $\phi_{x+1,0}\left(0 \times I_{1}\right)$ :

$$
\phi_{x+1,0}\left(0, w_{1}^{(1)}\right)=\phi_{x+1, x}(P) \in \mathrm{II},
$$

since, if $w=\frac{1}{2}, w^{\prime}>0$. Similarly, $\phi_{x+1,0}\left(0, w_{2}^{(1)}\right) \in \mathrm{VI}$, since, if $w=0, w^{\prime}<0$. Thus a piece $P_{1} Q_{1}$ of $\phi_{x+1,0}\left(0 \times I_{1}\right)$ lies in IV and

$$
P_{1} \in\left\{(v, w) \mid v>0, w=\frac{1}{2}\right\}, \quad Q_{1} \in\{(v, w) \mid v>0, w=0\} .
$$

This argument can be repeated, the result being that there exist closed intervals $I_{0} \supset I_{1} \supset I_{2} \supset \ldots$, such that $\phi_{x+n, 0}\left(0 \times I_{n+1}\right)$ lies in $\overline{I V}$. The set

$$
\bigcap_{n=0}^{\infty} I_{n}
$$

contains (at least) an initial condition with the desired properties.

The proof of Theorem 3.2 is finished. In the rest of this section we prove that, as $d \uparrow d_{n}$, the fixed point $u_{2 n}^{*}$ goes to zero pointwise in $x$. We write the proof explicitly only for the case $n=2$. It is sufficient to show that for $d=4-\varepsilon$ there exists a $\delta(\varepsilon)>0$ such that $\delta(\varepsilon) \rightarrow 0$ as $\varepsilon \downarrow 0$ and that the solution of (4.4) with $v(0)=0$ and $w(0)=-\delta(\varepsilon)$ blows up in finite time going successively through regions V, III, IV, II. This refinement of Proposition 4.2 can then be used with Proposition 4.3 to give (by the same Bleher-Sinai argument of Proposition 4.4) a global solution of (4.4) with

$$
-\delta(\varepsilon) \leqq w(0) \leqq w_{2}(\varepsilon), \quad v(0)=0,
$$

where $w_{2}(\varepsilon)$ is the constant appearing in Proposition 4.3.

The idea to prove the existence of the function $\delta(\varepsilon)$ is a perturbative argument to control the solution until it enters region IV together with a monotonicity argument to compare solutions of the non-linear equation to solutions of the linearized equation. Consider first the case $d=4(\sigma=3)$. We show that all solutions with initial conditions $(0,-\delta)$ and $\delta$ sufficiently small blow up in finite time going through V, III, IV, II. To set up perturbation theory we rescale (4.4), $v \rightarrow \delta v, w \rightarrow \delta w$ :

$$
v^{\prime}=w, \quad w^{\prime}=\frac{x}{\sigma} w-v+\delta 2 v w .
$$

Let $\phi_{x_{2}, x_{1}}^{(\delta)}$ denote the flow of (4.23). For $\delta=0, \phi_{x_{2}, x_{1}}^{(0)}$ is linear and given by

$$
\phi_{x_{2}, x_{1}}^{(0)}=\phi_{x_{2}, 0}^{(0)}\left(\phi_{x_{1}, 0}^{(0)}\right)^{-1}, \quad \phi_{x, 0}^{(0)}=\left(\begin{array}{cc}
\tilde{U}_{\sigma}(x) & \tilde{V}_{\sigma}(x) \\
\tilde{U}_{\sigma}^{\prime}(x) & \tilde{V}_{\sigma}^{\prime}(x)
\end{array}\right),
$$

where

$$
\tilde{U}_{\sigma}(x)=U_{\sigma}\left(\sigma^{-1 / 2} x\right), \quad \tilde{V}_{\sigma}(x)=\sigma^{1 / 2} V_{\sigma}\left(\sigma^{-1 / 2} x\right)
$$


and $U_{\sigma}, V_{\sigma}$ are given in the appendix. First order perturbation theory gives

$$
\begin{aligned}
\phi_{x, 0}^{(\delta)}\left(\begin{array}{c}
0 \\
-1
\end{array}\right) & =\phi_{x, 0}^{(0)}\left(\begin{array}{c}
0 \\
-1
\end{array}\right)+\delta \int_{0}^{x} \phi_{x, y}^{(0)}\left(\begin{array}{c}
0 \\
2 \tilde{V}_{\sigma}^{\prime}(y) \tilde{V}_{\sigma}^{\prime}(y)
\end{array}\right) d y+O\left(\delta^{2}\right) \\
& =\phi_{x, 0}^{(0)}\left(\begin{array}{c}
\delta \int_{0}^{x} e^{-y^{2} / 2 \sigma}\left(-2 \widetilde{V}_{\sigma}(y)^{2} \widetilde{V}_{\sigma}^{\prime}(y)\right) d y \\
-1+O(\delta)
\end{array}\right)+O\left(\delta^{2}\right) .
\end{aligned}
$$

If $\sigma=3, \widetilde{V}_{3}(y)=-y^{3} / 9+y$ and

$$
-2 \delta \int_{0}^{\infty} e^{-y^{2} / 6} \tilde{V}(y)^{2} \tilde{V}^{\prime}(y) d y=12 \sqrt{6 \pi}>0 .
$$

Choose $x_{1}>0$ so large that $-\tilde{V}_{3}\left(x_{1}\right)>0$ and

$$
a\left(x_{1}\right) \equiv-2 \int_{0}^{x_{1}} e^{-y^{2} / 6} \tilde{V}^{2} \tilde{V}^{\prime} d y>0 .
$$

Assume also $x_{1}>6$ for later purposes. Let furthermore

$$
z^{(0)}(x)=\phi_{x, x_{1}}^{(0)} \circ \phi_{x_{1}, 0}^{(\delta)}\left(\begin{array}{c}
0 \\
-1
\end{array}\right), \quad x \geqq x_{1}, \quad z(x)=\phi_{x, 0}^{(\delta)}\left(\begin{array}{c}
0 \\
-1
\end{array}\right) .
$$

The preceding argument implies that

$$
z^{(0)}(x)=(-1+O(\delta))\left(\begin{array}{c}
\tilde{V}_{3} \\
\tilde{V}_{3}^{\prime}
\end{array}\right)+\left(a\left(x_{1}\right) \delta+O\left(\delta^{2}\right)\right)\left(\begin{array}{c}
\tilde{U}_{3} \\
\tilde{U}_{3}^{\prime}
\end{array}\right)
$$

But, by the results in the appendix, $\widetilde{V}_{3}, \widetilde{V}_{3}^{\prime} \rightarrow-\infty$ and $\tilde{U}_{3}, \widetilde{U}_{3}^{\prime} \rightarrow+\infty$, as $x \rightarrow \infty$ and if $\delta$ is small enough (depending on $x_{1}$ )

$$
z^{(0)}(x) \rightarrow\left(\begin{array}{c}
+\infty \\
+\infty
\end{array}\right), \quad x \rightarrow \infty
$$

Let now $\Delta z=z-z^{(0)}=\left(\begin{array}{l}\Delta v \\ \Delta w\end{array}\right)$. We claim that

$$
\Delta v \geqq 0, \quad \Delta w \geqq 0, \text { for all } x \geqq x_{1} .
$$

An immediate consequence of (4.30) is that $z(x)$ blows up since it must enter the (rescaled) region $\mathrm{II}=\{(v, w) \mid v \geqq 0, w \geqq 1 / 2 \delta\}$. The claim is proven by noting that the set

$$
S=\{(\Delta v, \Delta w) \mid \Delta w \geqq \Delta v \geqq 0\}
$$

contains $0=\left(\Delta v\left(x_{1}\right), \Delta w\left(x_{1}\right)\right)$ and is invariant if $x \geqq x_{1}$. Indeed, in $S$

$$
\frac{d}{d x}(\Delta w-\Delta v) \geqq \frac{x_{1}}{3} \Delta w-\Delta v-\Delta w \geqq \Delta w-\Delta v, \quad \frac{d}{d x} \Delta v \geqq \Delta w \geqq 0,
$$

where we used $x_{1} \geqq 6$ and the negativity of the non-linearity $-2 v w$.

For each $\delta>0$ sufficiently small we can thus choose a time $x_{2}(\delta)$ such that $z\left(x_{2}\right)$ is in the interior of region II. If $\varepsilon=4-d>0$ is small depending on $\delta$ the solution of (4.4) with initial condition $(0,-\delta)$ still has the property of being in region II after 
$x_{2}$. This defines a function $\varepsilon(\delta) \rightarrow 0$ as $\delta \rightarrow 0$ which is the inverse of the desired function $\delta(\varepsilon)$.

\section{The Non-Trivial $\varphi^{2 n}$ Fixed Points}

In this section, we prove the existence of all $\varphi^{2 n}$ fixed points (Theorem 3.2). In the preceding section we have proven only the part of Proposition 4.1 which is relevant to the construction of the $\varphi^{4}$ fixed point. We first complete the proof of Proposition 4.1, and then construct a solution which winds around the origin $n-1$ times if $2<d<d_{n}$, a generalization of Proposition 4.3. We eventually construct the fixed point, replacing the Bleher-Sinai argument by a slightly more abstract one.

Proof of Proposition 4.1 (continued). What is left to prove is V $\rightarrow$ III in the case where $x_{0}>0$. It is convenient to change variables: define $s=v / x ;(4.4)$ becomes

$$
x s^{\prime}=w-s, \quad w^{\prime}=x\left(\frac{1}{\sigma} w-s+2 s w\right) .
$$

Region $\mathrm{V}$ is then further partitioned in three parts $\mathrm{V}_{1}, \mathrm{~V}_{2}, \mathrm{~V}_{3}$ [note that $(v, w) \in \mathrm{V} \Leftrightarrow(s, w) \in \mathrm{V}]$ :

We prove that

$$
\begin{aligned}
& \mathrm{V}_{1}=\{(s, w) \in \mathrm{V} \mid w \geqq s\}, \\
& \mathrm{V}_{2}=\left\{(s, w) \in \mathrm{V} \mid s>w \geqq \frac{2 \sigma s}{1+2 \sigma s}\right\}, \\
& \mathrm{V}_{3}=\left\{(s, w) \in \mathrm{V} \mid w<\frac{2 \sigma s}{1+2 \sigma s}\right\} .
\end{aligned}
$$

$$
\mathrm{V}_{3} \rightarrow \mathrm{V}_{2} \rightarrow \mathrm{V}_{1} \rightarrow \mathrm{III}
$$

in the notation of Proposition 4.1. Note first that on the boundary $\mathrm{V}_{12}=\{s=w\} \cap V$, $s^{\prime}=0$, and $w^{\prime}>0$, so that the vector field points into region $V_{1}$ and this boundary can only be crossed in one direction. Similarly, on $\mathrm{V}_{23}=\left\{w=2 \sigma s(1+2 \sigma s)^{-1}\right\} \cap V$, $w^{\prime}=0, s^{\prime}<0$, so that also $\mathrm{V}_{23}$ has this property. The arguments to prove (5.2) are very similar to the ones used in Sect. 4 to prove Proposition 4.1. Therefore we will be rather sketchy. We always assume $x_{0}>0$.

(a) $\mathrm{V}_{1} \rightarrow$ III: In $\mathrm{V}_{1}, w \leqq 0$ implies that

$$
s^{\prime} \leqq-\frac{s}{x} \Rightarrow s \leqq s_{0} \frac{x_{0}}{x} .
$$

Using this and $w \geqq s$ we conclude that in $\mathrm{V}_{1}$,

$$
w^{\prime} \geqq-\left(1-\sigma^{-1}\right) x s \geqq-\left(1-\sigma^{-1}\right) x_{0} s_{0},
$$

since $\sigma>0$. Thus $w$ becomes positive within finite time.

(b) $\mathrm{V}_{2} \rightarrow \mathrm{V}_{1}$ : In $\mathrm{V}_{2}, w-s$ is negative but increasing

$$
\frac{d}{d x}(w-s) \geqq-\frac{(w-s)}{x} .
$$


It follows that after a time $x_{1}=x_{1}\left(w_{0}, s_{0}, y_{0}, \varepsilon\right), w-s \geqq \varepsilon$. For $x>x_{1}$,

$$
w^{\prime} \geqq x\left(\frac{w}{\sigma}-s\right) \geqq x_{1}\left[\left(\sigma^{-1}-1\right) s_{0}-\varepsilon / \sigma\right],
$$

which is bounded below by a positive constant if $\varepsilon$ is small enough. This bound together with $s^{\prime}>0$ implies the claim.

(c) $\mathrm{V}_{3} \rightarrow \mathrm{V}_{2}: w^{\prime} \leqq 0 \Rightarrow w \leqq w_{0}$. We then use the bound defining $\mathrm{V}_{3}$ :

$$
s^{\prime} \leqq \frac{1}{x} \min \left(\frac{\sigma s}{1+2 \sigma s}-s, w_{0}-s\right) \leqq \frac{1}{x} \min \left((\sigma-1) s, w_{0}-s\right)
$$

implying that after finite time, $(s, w)$ enters $\mathrm{V}_{2}$.

As in Sect. 4, we modify the equation, this time only in region II, to avoid solutions which blow up:

$$
\begin{gathered}
v^{\prime}=w, \\
w^{\prime}=\left\{\begin{array}{ll}
\frac{x}{\sigma} w-v+2 v w & (v, w) \notin \mathrm{II} \\
\frac{x}{\sigma} w & (v, w) \in \mathrm{II}
\end{array} .\right.
\end{gathered}
$$

Again this modification does not affect solutions which do not enter II. We consider now solutions with initial conditions (at $x=0$ ) on the $w$-axis and define a winding number as the number of times the solution winds around the origin until it crosses a coordinate axis for the last time. In formula, let $(v, w) \neq(0,0)$ be a solution of (5.3) with $v(0)=0$, and $\operatorname{tg} \varphi=\frac{w}{v}$. The winding number,

$$
N(v, w)=\frac{1}{2}\left[-\frac{1}{\pi} \int_{0}^{\infty} \frac{d \varphi}{d x} d x\right], 2
$$

is well defined since $\frac{d \varphi}{d x}=\left(v^{2}+w^{2}\right)^{-1}\left(w v^{\prime}-v w^{\prime}\right)$ is continuous and finite. Since, by Proposition 4.1, all solutions with finite winding number eventually stay in II $\cup$ IV, we see that $N \in \mathbb{N}$ if $w(0)>0$ and $N \in \mathbb{N}+\frac{1}{2}$ if $w(0)<0$. The $\varphi^{4}$ fixed point constructed in Sect. 4 has $N=\frac{1}{2}$. Theorem 3.2 can be restated as follows: if $2 n-1$ $<\sigma<\infty, n=2,3, \ldots$, there exists a solution $\left(v_{2 n}^{*}, w_{2 n}^{*}\right)$ of $(5.3)$, with $v_{2 n}^{*}(0)=0$, not entering region II, with winding number

$$
N\left(v_{2 n}^{*}, w_{2 n}^{*}\right)=\frac{n-1}{2} .
$$

Assume for definiteness that $n$ is odd. The even case can be dealt with the same way. We want to construct a solution of (5.3) with integer winding number and initial condition $\left(0, w_{0}>0\right)$ at $x=0$. View $N(\cdot)$ as a map from the set $\mathbb{R}_{+}=\left\{w_{0} \mid w_{0}>0\right\}$, with the induced topology, to the integers. This map has the following properties:

${ }^{2}$ Here, [ ] means integer part 
Proposition 5.1. Let $\phi_{x, y}$ be the flow of (5.3).

(i) $\left\{w_{0} \in \mathbb{R}_{+} \mid N\left(w_{0}\right)=N\right.$ and $\phi_{x, 0}\left(0, w_{0}\right) \in$ II for some $\left.x\right\}$ is open for $N \in \mathbb{N}$.

(ii) $\left\{w_{0} \in \mathbb{R}_{+} \mid N\left(w_{0}\right) \leqq N\right\}$ is closed for all $N \in \mathbb{N}$.

(iii) If $w_{1}^{(n)}, w_{2}^{(n)}$ are two sequences converging to $w_{0}$, then

$$
\left|\lim _{n \rightarrow \infty} N\left(w_{1}^{(n)}\right)-\lim _{n \rightarrow \infty} N\left(w_{2}^{(n)}\right)\right| \leqq 1 .
$$

Proof. (i) II is invariant, and the vector field on its boundary does not vanish and points to the interior of II. Thus if $\phi_{x, 0}\left(0, w_{0}\right) \in \mathrm{II}$ for some $x$, then for any $x_{1}>x$, $\phi_{x_{1}, 0}\left(0, w_{0}\right)$ is in the interior of II. By continuity of $\phi$, if $|\delta|$ is small enough, also $\phi_{x_{1}, 0}\left(0, w_{0}+\delta\right) \in \mathrm{II}$.

(ii) Let $w_{0} \in \mathbb{R}_{+}$be an accumulation point of $w_{N}=\left\{w_{0} \in \mathbb{R}_{+} \mid N\left(w_{0}\right) \leqq N\right\}$. Let us suppose that $N\left(w_{0}\right) \geqq N+1$ and derive a contradiction. The solution $\phi_{x, 0}\left(0, w_{0}\right)$ winds around the origin $N$ times ending up in region II $\cup$ IV and then crosses the $v$-axis to begin the next wind. At some time $x_{1}$ the solution is in the interior of region VI after $N$ turns. But by continuity, initial conditions in a whole neighborhood of $w_{0}$ give solutions which share this property at $x=x_{1}$, which is a contradiction, since all these solutions have winding number $>N$.

(iii) Let $N_{i}=\lim _{n \rightarrow \infty} N\left(w_{i}^{(n)}\right), i=1,2$. We can assume that $N_{1}<N_{2}$ and, by (ii), $N\left(w_{0}\right)=N_{1}$. First note that, if $(v(x), w(x))=\phi_{x, 0}\left(0, w_{0}\right), \lim _{x \rightarrow \infty} v(x)=\infty$, since the only possibility for $v$ to stay bounded in II $\cup$ IV is that $w(x)=v^{\prime}(x) \rightarrow 0$ as $x \rightarrow \infty$, i.e. that $(v, w)$ converge to a fixed point on the positive $v$ axis, which does not exist. Let $A$ be a large positive number to be chosen later. Let $x_{1}$ be so large that $(v(x), w(x))$ winds around the origin $N_{1}$ times for $0 \leqq x \leqq x_{1}$ and $v\left(x_{1}\right) \geqq A$. If $n_{0}$ is large enough and $n \geqq n_{0}$, also $\left(v^{(n)}(x), w^{(n)}(x)\right)=\phi_{x, 0}\left(0, w_{2}^{(n)}\right)$ winds $N_{1}$ times up to time $x_{1}$ and $v^{(n)}\left(x_{1}\right)$ $\geqq A / 2$. Since (for $n$ large) $N\left(w_{2}^{(n)}\right)=N_{2}$ there exists an $x_{2} \geqq x_{1}$ (depending on $n$ ) such that $w^{(n)}\left(x_{2}\right)=0$ and $w^{(n)}(x)>0$ for $x_{1}<x<x_{2}$. In II $\cup \mathrm{IV}, v^{\prime}=w \geqq 0$, so that $v^{(n)}\left(x_{2}\right) \geqq \frac{A}{2}$. The argument in the proof of Proposition 4.2 can be used: choose $A$ so that $J(A / 2,0)=J\left(0, w_{1}\right)$, where $w_{1}=w_{1}(\sigma)$ is the constant defined in Proposition 4.2. Since $J$ is increasing $\left(v^{(n)}, w^{(n)}\right)$ will cross the negative $w$ axis below $w_{1}(\sigma)$, and will enter the invariant region II doing $N_{1}+1$ turns around the origin.

The proof of Theorem 3.2 is now simple: fix $\sigma \in] 2 n-1, \infty$ [ for $n$ odd $\geqq 2$. The function $N: \mathbb{R}_{+} \rightarrow \mathbb{N}$ takes the value zero [if $w_{0}>1 / 2, N\left(w_{0}\right)=0$ ] and a value $\geqq(n+1) / 2$ [if $w_{0}$ is small enough so that the linear analysis in the appendix is applicable, one constructs as in Proposition 4.2 a solution with $\left.N\left(w_{0}\right) \geqq(n-1) / 2\right]$. By (iii) in Proposition 5.1, $N(\cdot)$ has discontinuities of at most one and thus takes all integer values between 0 and $(n+1) / 2$. Hence there are (at least) $(n-1) / 2$ discontinuity points $w_{1}, \ldots, w_{(n-1) / 2} \in \mathbb{R}_{+}$such that

$$
\varliminf_{w \rightarrow w_{m}} N(w)=\varlimsup_{w \rightarrow w_{m}} N(w)-1=m \text {. }
$$

By the lower semicontinuity property (ii), $N\left(w_{m}\right)=m$. But by (i), $\phi_{x, 0}\left(0, w_{m}\right) \notin \mathrm{II}$, for all $x \geqq 0$, and thus $\phi_{x, 0}\left(0, w_{m}\right)$ is also a global solution of the unmodified equation (4.4) with winding number $m$, and the proof of Theorem 3.2 when $m$ is odd is 
complete. If $n$ is even, note that $N: \mathbb{R}_{-} \rightarrow \mathbb{N}+\frac{1}{2}$ takes the value $\frac{1}{2}$ by Proposition 4.2 and a value $\geqq(n+1) / 2$ by linear analysis. Then proceed exactly as in the odd case.

\section{Appendix}

In this appendix we count the zeros of the solutions of the linearization of (4.3). After changing variable $x \rightarrow x \sqrt{\sigma}$ the equation becomes:

$$
u^{\prime \prime}-z u^{\prime}+\sigma u=0 \text {. }
$$

Let $U_{\sigma}(z)$ and $V_{\sigma}(z)$ be the solutions of (A1) with data

$$
U_{\sigma}(0)=1, \quad U_{\sigma}^{\prime}(0)=0, \quad V_{\sigma}(0)=0, \quad V_{\sigma}^{\prime}(0)=1 .
$$

These functions can be represented by the integrals

$$
\begin{aligned}
& U_{\sigma}(z)=\frac{2^{1+\sigma / 2}}{\Gamma(-\sigma / 2)} \int_{0}^{\infty} \cosh z t e^{-t^{2} / 2} t^{-\sigma-1} d t, \quad \operatorname{Re} \sigma<0, \\
& V_{\sigma}(z)=\frac{2^{(1+\sigma) / 2}}{\Gamma(-(\sigma-1) / 2)} \int_{0}^{\infty} \sinh z t e^{-t^{2} / 2} t^{-\sigma-1} d t, \quad \operatorname{Re} \sigma<1,
\end{aligned}
$$

and can also be expressed in terms of parabolic cylinder functions. To compute $U_{\sigma}, V_{\sigma}$ outside the range of validity of (A3) one can use the recursions,

$$
U^{\prime}(z)=-\sigma V_{\sigma-1}(z), \quad V_{\sigma}^{\prime}(z)=U_{\sigma-1}(z),
$$

whose proof is based on the fact that if $u$ is a solution of (A1) then $u^{\prime}$ is a solution of (A1) with $\sigma$ replaced by $\sigma-1$.

Proposition. Let $U_{\sigma}, V_{\sigma}$ be the solutions of (A1) with data (A2). Then $U_{\sigma}$ has no zero if $\sigma \leqq 0$, and exactly $2 n$ zeros if $2 n-2<\sigma \leqq 2 n, n \geqq 1 ; V_{\sigma}$ has exactly one zero if $\sigma \leqq 1$ and exactly $2 n-1$ zeros if $2 n-3<\sigma \leqq 2 n-1, n \geqq 1$.

Proof. The facts that $U_{\sigma}$ is positive if $\sigma<0$ and that $V_{\sigma}(z)>0$ for $z>0$ if $\sigma<1$ follow from the integral representation (A3). From (A4) we deduce that $U_{0}(z)=1$, $V_{1}(z)=z$. Next we prove inductively:

(i) If $2 n-2<\sigma \leqq 2 n, n \geqq 0$, then $U_{\sigma}(z)$ has exactly $n$ positive zeros $0<z_{1}<\ldots<z_{n}$; $\operatorname{sign} U_{\sigma}^{\prime}\left(z_{i}\right)=(-1)^{i}$ and, as $z \rightarrow \infty, U_{\sigma}(z) \rightarrow(-1)^{n} \infty$, except for $\sigma=0$.

(ii) If $2 n-3<\sigma \leqq 2 n-1, n \geqq 0$, then $V_{\sigma}(z)$ has exactly $n$ nonnegative zeros $0=z_{1}<\ldots<z_{n} ; \operatorname{sign} V_{\sigma}^{\prime}\left(z_{i}\right)=(-1)^{i}$ and, as $z \rightarrow \infty, V_{\gamma}(z) \rightarrow(-1)^{n-1} \infty$.

These properties are readily checked for $n=0$. We first prove (ii) for $n=N+1$ assuming (i) for $n=N$. Let $2(N+1)-3<\sigma \leqq 2(N+1)-1$ and let $0<z_{1}<\ldots<z_{N}$ be the positive zeros of $U_{\sigma-1}$. For $0<z<z_{1}, V_{\sigma}^{\prime}>0$, and, since $V_{\sigma}^{\prime}(0)=1, V(z)>0$. From $V_{\sigma}^{\prime}\left(z_{i}\right)=U_{\sigma-1}\left(z_{i}\right)=0$ and $\operatorname{sign} V_{\sigma}^{\prime \prime}\left(z_{i}\right)=\operatorname{sign} U_{\sigma-1}^{\prime}\left(z_{i}\right)=(-1)^{i}$, it follows, using (A1), that $\operatorname{sign} V_{\sigma}\left(z_{i}\right)=(-1)^{i+1}$. Thus between two consecutive zeros $z_{i}, z_{i+1}$ of $U_{\sigma-1}$ there is a zero $\bar{z}_{i+1}$ of $V_{\sigma}$. This zero is unique and $V_{\sigma}^{\prime}\left(\bar{z}_{i+1}\right) \neq 0$, since $V_{\sigma}^{\prime}(z)$ has a definite sign in $] z_{i}, z_{i+1}\left[\right.$. Similarly, $(-1)^{N} U_{\sigma-1}(z)>0$ for $z>z_{N}$, and $U_{\sigma-1}(z)$ $\rightarrow(-1)^{N} \infty(z \rightarrow \infty)$ imply $V_{\sigma}(z) \rightarrow(-1)^{N} \infty$ monotonically for $z_{N}<z \rightarrow \infty$. But since $\operatorname{sign} V_{\sigma}\left(z_{N}\right)=(-1)^{N+1}$, there must be exactly one zero of $V_{\sigma}$ in the interval $] z_{N}, \infty[$ 
making a total of $N+1$ zeros. In the same way one proves that (ii) for $n=N$ implies (i) for $n=N+1$, and the proof is complete.

Acknowledgements. I wish to thank C. Albanese, G. Gallavotti, K. Gawedzki, T. Kennedy, J. Lott, and T. Spencer for useful discussions and remarks, as well as B. Tirozzi for assistance in a numerical study of the differential equation.

\section{References}

1. Wilson, K.G.: Renormalization group and critical phenomena. I, II. Phys. Rev. B 4, 3174-3183 and 3184-3205 (1971)

Wilson, K.G., Kogut, J.B.: The renormalization group and the $\varepsilon$-expansion. Phys. Rep. 12, 76-199 (1974)

2. Wilson, K.G., Fisher, M.E.: Critical exponents in 3.99 dimensions. Phys. Rev. Lett. 28, 240-243 (1972)

3. Dyson, F.: Existence of a phase transition in a one-dimensional Ising ferromagnet. Commun. Math. Phys. 12, 91-107 (1969); An Ising ferromagnet with discontinuous long range order. Commun. Math. Phys. 21, 269-283 (1971)

4. Bleher, P.M., Sinai, Ya.G.: Investigation of the critical point in models of the type of Dyson's hierarchical model. Commun. Math. Phys. 33, 23-42 (1973)

5. Collet, P., Eckmann, J.-P.: The $\varepsilon$-expansion for the hierarchical model. Commun. Math. Phys. 55, 67-96 (1977); A renormalization group analysis of the hierarchical model in statistical mechanics. Lecture Notes in Physics, Vol. 74. Berlin, Heidelberg, New York: Springer 1978

6. Gawedzki, K., Kupiainen, A.: Non-Gaussian fixed points of the block spin transformation. Hierarchical model approximation. Commun. Math. Phys. 89, 191-200 (1983)

7. Koch, H., Wittwer, P.: A non-gaussian renormalization group fixed point for hierarchical scalar lattice field theories. Commun. Math. Phys. 106, 495-532 (1986)

8. Gallavotti, G.: Some aspects of the renormalization problems in statistical mechanics and field theory. Memorie dell'Accademia dei Lincei, S. 8, Vol. 15, 1978

9. Hasenfratz, A., Hasenfratz, P.: Renormalization group study of scalar field theories. Nucl. Phys. B 270 [FS 16], 687-701 (1986)

10. Brydges, D.C., Kennedy, T.: Mayer expansions and Burger's equation. University of Virginia preprint, 1986

Brydges, D.C.: Expansions in statistical mechanics as part of the theory of partial differential equations. University of Virginia preprint 1986

11. Wilson, K.G., Kogut, J.B.: Ref. [1, Chap. 11]

12. Wegner, F.J.: The critical state, general aspects. In: Phase transitions and critical phenomena, Vol. VI. Domb, C., Green, M.E. (eds.). New York: Academic Press 1976

13. Giga, Y., Kohn, R.V.: Asymptotically self-similar blow-up of semilinear heat equation. Commun. Pure Appl. Math. 38, 297-319 (1985)

14. Felder, G.: Non-trivial renormalization group fixed points. Preprint IHES/P/86/48, to appear in the proceedings of the IAMP conference, Marseille 1986

15. Polchinski, J.: Renormalization of effective Lagrangians. Nucl. Phys. B 231, 269-295 (1984)

Communicated by K. Gawẹdzki

Received December 11, 1986; in revised form January 14, 1987 
